B. Laits de petit mélange (Bídons de 20 à 10 litres).

\begin{tabular}{|c|c|c|c|c|c|c|c|c|}
\hline Bidons Nos & $\begin{array}{l}\text { Densité } \\
\dot{a}+15^{\circ}\end{array}$ & $\begin{array}{c}\text { Extrait sec } \\
\dot{a}+100^{\circ} \\
\text { par litre }\end{array}$ & $\begin{array}{c}\text { Cendres } \\
\text { par litre }\end{array}$ & $\begin{array}{c}\text { Matière } \\
\text { grasse } \\
\text { par litre }\end{array}$ & $\begin{array}{c}\text { Caséine } \\
\text { par litre }\end{array}$ & $\begin{array}{l}\text { Lactose } \\
\text { anhydre } \\
\text { parlitre }\end{array}$ & $\begin{array}{c}\text { Chloru- } \\
\text { res } \\
\text { rar litre }\end{array}$ & $\begin{array}{c}\text { Extrait } \\
\text { dégr. } \\
\text { par litre }\end{array}$ \\
\hline 1 (19 litres) & $1.032,2$ & 113 gr. 60 & 6 gr. 81 & 24 gr. & $28 \mathrm{gr}$. & $52 \mathrm{gr} .45$ & 1 gr. 55 & 89,6 \\
\hline 2 (20 litres) & 1.031 & 119 gr. 35 & $7 \mathrm{gr}$. & $31 \mathrm{gr}$. & $30 \mathrm{gr}$. & 49 gr. 95 & 1 gr. 78 & 88,35 \\
\hline 3 (19 litres) & $1.031,2$ & 114. gr. 65 & $7 \mathrm{gr} .27$ & $23 \mathrm{gr}$. & 30 gr. 75 & 50 gr. 60 & 1 gr. 65 & 91,65 \\
\hline 4 (20 litres) & $1.031,6$ & 122 gr. 85 & $7 \mathrm{gr}$. & $28 \mathrm{gr}$. & $33 \mathrm{gr}$. & 51 gr. 75 & 1 gr. 70 & 94,85 \\
\hline 5 (20 litres) & $1.031,7$ & 122 gr. 80 & 7 gr. 19 & $25 \mathrm{gr}$. & $33 \mathrm{gr} .50$ & $53 \mathrm{gr} .95$ & 1 gr. 67 & 97,8 \\
\hline 6 (10 litres) & 1.031 & 119 gr. 48 & 7 gr. 12 & $28 \mathrm{gr}$. & $31 \mathrm{gr}$. & 51 gr. 30 & 1 gr. 80 & 91,5 \\
\hline 7 (20 litres) & $1.031,1$ & $123 \mathrm{gr}$. & 7 gr. 05 & $31 \mathrm{gr}$. & $32 \mathrm{gr}$. & $51 \mathrm{gr} .55$ & 1 gr. 72 & 92,0 \\
\hline 8 (20 litres) & $1.030,2$ & 107 gr. 50 & 7 gr. 70 & $21 \mathrm{gr}$. & 29 gr. 25 & 47 gr. 20 & 2 gr. 09 & 86,5 \\
\hline 9 (19 litres) & 1.032 & $114 \mathrm{gr} .45$ & 6 gr. 90 & $23 \mathrm{gr}$. & $31 \mathrm{gr}$. & 50 gr. 40 & 1 gr. 70 & 91,45 \\
\hline 10 (20 litres) & $1.031,2$ & 122 gr. 70 & 6 gr. 60 & $30 \mathrm{gr}$. & $33 \mathrm{gr}$. & $49 \mathrm{gr} .75$ & 1 gr. 72 & 92,7 \\
\hline 11 (20 litres) & $1.030,4$ & 117 gr. 82 & 7 gr. 06 & $27 \mathrm{gr}$. & 30 gr. 75 & $50 \mathrm{gr} .85$ & 2 gr. 05 & 90,80 \\
\hline 12 (20 litres) & $1.031,1$ & 115 gr. 60 & 7 gr. 20 & $26 \mathrm{gr}$. & 30 gr. 75 & 49 gr. 50 & 1 gr. 90 & 89,6 \\
\hline 13 (20 litres) & $1.032,3$ & $120 \mathrm{gr}$. & 7 gr. 20 & $25 \mathrm{gr} .50$ & 33 gr. 50 & 49 gr. 75 & 1 gr. 82 & 94,5 \\
\hline 14 (19 litres) & $1.030,2$ & 118 gr. 80 & $7 \mathrm{gr} .65$ & $28 \mathrm{gr}$ & $31 \mathrm{gr}$. & 49 gr. 30 & 2 gr. 10 & 90,8 \\
\hline 15 (15 litres) & $1.031,3$ & 145 gr. 10 & $7 \mathrm{gr} .55$ & $43 \mathrm{gr}$. & $39 \mathrm{gr}$. & $51 \mathrm{gr}$. & 1 gr. 58 & 102,1 \\
\hline
\end{tabular}

C. Ensemble de la production totale de la traite.

Densité à $+15^{\circ} \ldots \ldots \ldots \ldots \ldots \ldots \ldots$

Extrait sec à $+100^{\circ} \ldots \ldots \ldots \ldots \ldots \ldots$

Cendres $\ldots \ldots \ldots \ldots \ldots \ldots \ldots \ldots \ldots$

Matière grasse $\ldots \ldots \ldots \ldots \ldots \ldots \ldots \ldots$.

Caséine ......................

Lactose anhydre.$\ldots \ldots \ldots \ldots \ldots \ldots$

Chlorures ......................

Extrait dégraissé $\ldots \ldots \ldots \ldots \ldots \ldots \ldots$

118 gr. 25 par litre

7 gr. 40 " "

27 gr. " "

30 gr. 75 "

49 gr. 30 " "

1 gr. 90 " "

91 gr. 25 .

(A suivre).

\title{
DOSAGE COLORIMÉTRIQUE DES PHOSPHATES DANS LE LAIT
}

\author{
par M. le Professeur Dr ANTolin PEÑA, \\ Chef de laboratoire de l'Institut de Puériculture de GiJon
}

Les méthodes décrites jusqu'à ces derniers temps pour déterminer les phosphates dans le lait, sont en général assez peu pratiques; leur technique longue et compliquée rend leur usage très peu répandu actuellement dans la pratique courante. Le dosage des phosphates dans le lait serait cependant d'une utilité incontestable pour déceler le mouillage et dépister les mammites tuberculeuses.

L'adaptation de la méthode de Copaux, faite par KuING et 
LASSIEUR, pour le dosage de l'acide phosphorique dans le lait (1), nous semble assez accessible à la pratique quotidienne, mais l'appréciation de ses résultats nous paraît sujette à d'assez graves erreurs. Nous avons préféré faire une application de la méthode de BELL et Dorsy (2) pour déterminer le phosphore dans le sang, car nous croyons que cette méthode colorimétrique réunit de grands avantages : la sensibilité, la faible quantité de réactifs à utiliser et la facile préparation de ceux-ci. Avec la technique que nous allons décrire, le dosage peut se faire en un temps très court, et, de plus, la détermination peut se faire en série sur plusieurs échantillons de lait.

Le principe de la méthode repose sur la coloration bleue qui se produit, dans un milieu alcalin, par réduction de l'acide phosphorique en présence d'une solution sulfurique de molybdate d'ammoniaque. Dans le phosphate de molybdène formé, la base est réduite partiellement, déterminant la formation de deux états d'oxygénation différents, qui produisent un bleu intense.

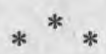

Nous utilisons les réactifs suivants :

$1^{0}$ Solution d'acide trichloracétique à $\ldots, \ldots \ldots \ldots, 20 \%$

$2^{\circ}$ Solution d'acide molybdique (3) :

Molybdate d'ammoniaque $\ldots \ldots \ldots \ldots \ldots \ldots . \quad 1 \mathrm{gr}$.

Acide sulfurique normal $\ldots \ldots \ldots \ldots \ldots \ldots, 20 \mathrm{~cm}^{3}$

Eau distillée Q. S. pour ............... $100 \mathrm{~cm}^{3}$ (3)

On dissout à chaleur douce le molybdate pulvérisé d'avance, dans l'acide sulfurique, puis on compète à $100 \mathrm{~cm}^{3}$.

$3^{\circ}$ Solution d'hydroquinone :

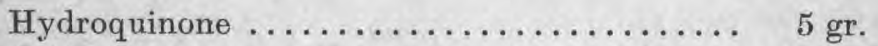

Acide sulfurique concentré .............. $0 \mathrm{~cm}^{3} 1$

Eau distillée Q. S. pour ............... $500 \mathrm{~cm}^{3}$

$4^{\circ}$ Solution de carbonate de sodium :

Solution A :

Carbonate de sodium

40 gr.

Eau distillée Q. S. pour .............. $200 \mathrm{~cm}^{3}$

(1) Annales des Falsifications et des Fraudes, avril 1923, n० 174, p. 141.

(2) Bell et Dorsy. Journ. of Biol. Chem. n० 174, p. 141, 1910, 44, 55.

(3) Pour vérifier, la pureté de la solution, mélanger $5 \mathrm{~cm}^{3}$ de ce réactif avec $5 \mathrm{~cm}^{3}$ de la solution d'hydroquinone et après 5 minutes de repos, ajouter $25 \mathrm{~cm}^{3}$ de la solution carbonatesulfite récemment préparée. Le tout doit rester parfaitement incolore. Cette vérification de la pureté des réactifs ne doit pas être négligée. 


\section{Solution B :}

Sulfite de sodium $\ldots \ldots \ldots \ldots \ldots \ldots \ldots \ldots, \quad 15$ gr.

Eau distillée Q. S. pour $\ldots \ldots \ldots \ldots \ldots \ldots, \quad 100 \mathrm{~cm}^{3}$

Au moment de l'emploi, faire le mélange de $20 \mathrm{~cm}^{3}$ de la solution A avec $5 \mathrm{~cm}^{3}$ de la solution $B$.

50 Solution-type (concentrée) :

Phosphate monopotassique pur et anhydre ... 4 gr. 394

Eau distillée .................... $\quad 1.000 \mathrm{gr}$.

$6^{\circ}$ Solution-type (diluée) :

On fait une dilution de la solution précédente de façon à la ramener à $5 \%$ et, pour faciliter sa conservation, on ajoute quelques gouttes de toluol. On ajoute de même du toluol pour conserver la solution concentrée.

Technique de l'opération. - Dans un matras gradué et contrôlé de $20 \mathrm{~cm}^{3}$, dans lequel on a versé $5 \mathrm{~cm}^{3}$ d'eau, on ajoute $2 \mathrm{~cm}^{3}$ de lait en mesurant très exactement. Après lavage de la pipette par trois fois dans de l'eau distillée, on ajoute $2 \mathrm{~cm}^{3}$ de la solution d'acide trichloracétique à $20 \%$ en agitant pendant le le mélange. On laisse reposer pendant 5 minutes, et on complète avec de l'eau jusqu'aux $20 \mathrm{~cm}^{3}$. Agiter et attendre encore pendant 10 minutes, après lesquelles on filtre sur un papier à filtrer préalablement lavé avec de l'acide nitrique et desséché à l'étuve pour éviter les traces de phosphore qu'il pourrait contenir. Ce liquide, parfaitement transparent, servira pour les déterminations.

Dans ce but, on prend des tubes parfaitement lavés à l'acide nitrique et à l'eau et desséchés à l'étuve, gradués à $10 \mathrm{~cm}^{3}$ (ce qui est très commode) ou non gradués. On utilise autant de tubes que l'on a d'échantillons de lait à étudier, avec un tube supplémentaire qui servira de témoin.

Dans le tube-témoin, on verse $0 \mathrm{~cm}^{3} 1$ de la solution d'acide trichloracétique et $1 \mathrm{~cm}^{3}$ de la solution-type diluée. Dans les tubes de recherches, on verse $1 \mathrm{~cm}^{3}$ du lait dilué au dixième, préparé comme il a été dit précédemment.

On verse alors dans tous les tubes $1 \mathrm{~cm}^{3}$ du réactif No 3 (solution d'hydroquinone) en agitant le mélange. Après 5 minutes, on ajoute $1 \mathrm{~cm}^{3}$ du mélange carbonate-sulfite en agitant de nouveau les tubes. Après 10 minutes, on verse dans le tube-témoin $6 \mathrm{~cm}^{3} 9$ d'eau distillée, et dans les autres tubes $7 \mathrm{~cm}^{3}$ (si les tubes sont gradués, il suffit de compléter jusqu'au trait supérieur). trique.

Après avoir agité de nouveau, on procède à la lecture colorimé-

La solution de phosphate monopotassique concentrée contient 
0 gr. 001 de phosphore par $\mathrm{cm}^{3}$; la solution diluée qui est utilisée en contient $0 \mathrm{gr}$. 00005. D'après ces données, et en se basant sur la formule générale colorimétrique, on emploie le facteur 2,29 pour obtenir le résultat en anhydride phosphorique. En ramenant au litre, on a :

$$
\mathrm{Q}=0,05 \times \frac{180}{\mathrm{a}} \times 2,29=\frac{11,45}{\mathrm{a}}
$$

Le quotient de 11,45 par la hauteur que le lait donne au colorimètre indique la quantité d'anhydride phosphorique par litre.

Nous avons utilisé pour nons rcherches le lait fourni à l'Institut. de Puériculture par deux fermes qui en assurent la fourniture journalière, et nous donnons ci-dessous les chiffres obtenus dans les cinquante déterminations faites.

\begin{tabular}{ll}
\multicolumn{2}{c}{ Ferme A } \\
\hline 1,01 & 1,02 \\
0,98 & 0,983 \\
1,02 & 1,102 \\
0,92 & 1,03 \\
0,89 & 1,05 \\
0,95 & 1,04 \\
1,01 & 1,02 \\
1,12 & 0,963 \\
0,96 & 0,971 \\
1,17 & 1,01 \\
1,05 & 1,03 \\
1,11 & 1,05 \\
1,02 &
\end{tabular}

\begin{tabular}{|c|c|}
\hline \multicolumn{2}{|c|}{ Ferme B } \\
\hline 1,17 & 1,01 \\
\hline 1,12 & 1,16 \\
\hline 1,14 & 1,23 \\
\hline 1,03 & 0,964 \\
\hline 1,26 & 0,988 \\
\hline 0,993 & 1,14 \\
\hline 1,04 & 1,02 \\
\hline 1,05 & 1,06 \\
\hline 1,15 & 1,09 \\
\hline 0,983 & 1,12 \\
\hline 1,06 & 1,19 \\
\hline 1,11 & 1,04 \\
\hline 1,16 & \\
\hline
\end{tabular}

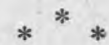

Nous nous sommes préoccupé de savoir si l'acide citrique et les citrates du lait provoquaient une modification de la réaction en milieu alcalin, et nous avons pour cela, en quelques cas, pratiqué, parallèlement avec nos recherches, la technique de BRIGGS en milieu acide(1) avec seulement l'emploi du sulfite de soude. En aucun cas nous n'avons trouvé des résultats différents. 